\title{
A two-dimensional triangular medical coding system for storing and reporting medical information.
}

\author{
Bandar Ali Suliman \\ College of Applied Medical Sciences, Taibah University, Madinah, Saudi Arabia
}

\begin{abstract}
Medical coding is an integral part of modern communication standards that help transform many types of clinical information such as patient information. The need to implement a standardized and a universal medical coding system is essential. The current study introduces a unique two-dimensional triangular medical coding system to identify, store and transmit clinical data based on the ICD-10 system between health institutions. The coding system comprises a triangular coding unit that can be arranged in six different standard geometric shapes representing the coding mechanism. A specific number of coding units are arranged in columns to form a cryptographic matrix. The matrix is comprised of many coding zones each generating a total number of "36" possible variations. The cryptographic code is then recognized by a digital scanner that is able to identify each triangular coding unit using a hypothetical signal triangle. The current coding system provides a simple process to store private medical information including patient's name, age, address, and medical history. This information can be then transferred electronically or printed without the fear of privacy or security violations.
\end{abstract}

Keywords: Medical coding, Triangular code, ICD 10.

Accepted on May 23, 2019

\section{Introduction}

Medical coding is an integral part of modern communication standards that help transform many types of clinical information such as patient information, history, current diagnosis, prescribed medication and other type of medically relevant data. For more than 50 years, medical scientists have been working to establish a standardized nomenclature process aimed at helping medical professionals in transcribing medical information in easy transferable codes [1].

The need to implement a standardized and a universal medical coding system that differs from the widely used onedimensional codes (barcodes) and two-dimensional codes (QR codes) is essential. Many researchers have found that using a manual entry system which requires a certain level of knowledge and competency may require additional validation and cross-checks to ensure information accuracy [2]. Automated solutions were then used to minimize these risks especially since the medically transcribed information was usually used by clinics and hospitals for financial and ensuring billing purposes [3].

Since the implementation of the International Statistical Classification of Diseases and Related Health Problems (ICD), Saudi Arabia was one of the first countries to start implementing the ICD-10 in its health information management systems [4]. The ICD-10 is the 10th revision of the medical classification system designed by the World Health
Organization (WHO) that was widely adopted since its completion in 1992 [5].

Many prior inventions and applications were specifically developed for the sole purpose of encoding medical information into unique identifiers either for patients [6] or for medical and clinical instruments [7]. The current project proposes a unique two-dimensional triangular medical coding system to identify, store and transmit clinical data based on the ICD-10 system in health institutions.

\section{Methods}

\section{The triangular coding system}

The triangular coding system comprises the main coding unit. The main coding unit is a single entity of two right-triangles where both are arranged so that the first triangle is placed on the second in a contiguous way sharing a single hypotenuse (Figure 1). One of right-triangles is white in colour, the other is black. Each angle of the two right-triangles points to the corners of a hypothetical square. The two right triangles can be arranged in six different standard geometric shapes representing the coding mechanism (Figure 2). Positions 1 and 6 show the presence of two right-triangles having the same colour; white and black respectively. Positions 2 through 4 show the presence of one right-triangle from each colour but in different geometrical rearrangements. 


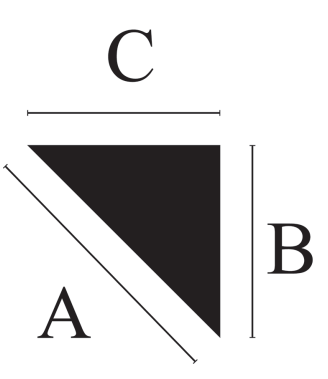

Figure 1. The main coding unit. Two right-triangles arranged so that the first triangle is placed on the second in a contiguous way sharing a single hypotenuse. A: shared hypotenuse; $B$ and $C$ : arms of the triangles.

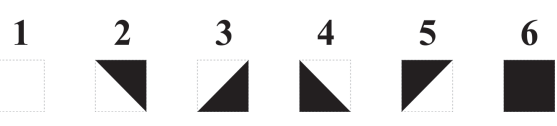

Figure 2. The variables generated by the main coding unit. Six different standard geometric shapes representing the variables generated by the main coding unit. Positions 1 and 6 show the presence of two right-triangles having the same color; white and black respectively. Positions 2 through 4 show the presence of one right-triangle from each colour but in different geometrical rearrangements.

\section{The cryptographic matrix}

The cryptographic matrix is created by forming columns of the main coding unit, each column consists of several main coding units. More than one column can be used; provided that the new column has the same number of main coding units as the preceding column (Figure 3).

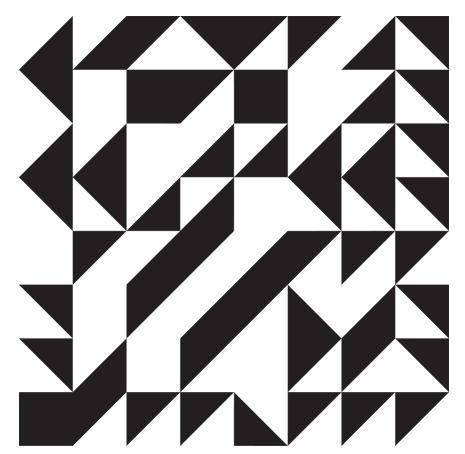

Figure 3. The Cryptographic matrix. A combination of columns where each column consists of a set number of main coding units the same as the column preceding it. In this example, each column consists of eight units.

\section{The coding zones}

The coding zone is a single entity constituting two adjacent main coding units. Since the total number of possible variables for a single main coding unit is six; the coding zones will generate a total number of " 36 " possible variations. The coding zones represent an alphanumerical system containing the single numbers from " 0 to " 9 " and the English alphabet from " $A$ " to "Z” (Figure 4).

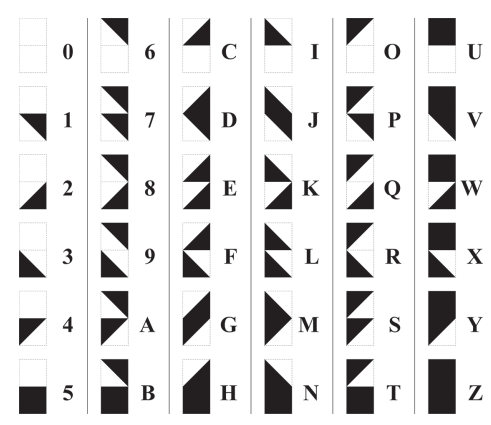

Figure 4. The Coding zones. Two main coding units constituting a single entity that can generate " 36 " possible variables. The variables are single alphanumerical characters; letters from " $A$ " to " $Z$ " and numbers from " 0 " and " 9 ".

\section{Matrix recognition}

The combination of cryptographic matrices is designed to encrypt information that belong to a certain dataset. The encrypted information requires a digital scanner to decrypt the code and extract viable information. The method by which a digital scanner is required to identify the main coding unit is a hypothetical triangle (the signal triangle) that determines where the signal of the scanner is directed. The signal triangle is positioned over the upper half of the hypothetical square (Figure 5) which was used to align the main coding unit. Each angle of the signal triangle will be scanned to transmit a binary signal; either " 0 " for negative or " 1 " for positive. Negative means a signal was not detected and therefore the white half of the main coding unit is identified. Positive means a signal was detected and therefore the black half of the main coding unit is identified. The outcome is a three-digit binary code for each main coding unit identifying the six possible rearrangements of the main coding unit (Table 1).

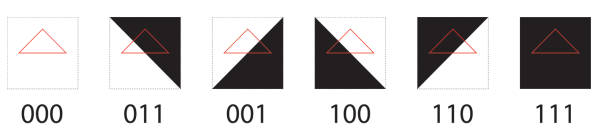

Figure 5. The signal triangle. A hypothetical triangle used to direct the signal from the digital scanner aiding the identification of the main coding unit. It is positioned over the upper half of the hypothetical square containing the two adjacent main coding units.

\section{Results and Discussion}

We report a triangular coding system that is able to store medical information based on the 10th revision of the International Statistical Classification of Diseases and Related Health Problems (ICD-10). The system relies on a basic coding unit that is composed of two right-angled triangles connected to each other and treated as a single unit where the head of each triangle points to a hypothetical square (Figure 1) and where the first triangle is positioned above the second triangle to form the main coding unit.

The coding information extracted from each of the major coding units is linked to thirty-six direct probabilities of single 
numbers from " 0 " to " 9 " and the English letters from " $\mathrm{A}$ " to "Z".

Table 1. The binary code resulting from the identification of the main coding unit.

\begin{tabular}{lllll}
\hline$\#$ & $\mathbf{X}$ & $\mathbf{Y}$ & $\mathbf{Z}$ & Code \\
\hline 1 & & & 000 \\
\hline 2 & & + & + & 011 \\
\hline 3 & & & + & 001 \\
\hline 4 & + & & 100 \\
\hline 5 & + & + & & 110 \\
\hline 6 & + & + & + & 111 \\
\hline
\end{tabular}

This is because the triangles used in the current study could be arranged into six different geometric forms (Figure 2). Since the main coding unit is two adjacent trajectories of a single legal entity, the output of mathematical exchanges can be measured using the following equation:

$\mathrm{P}=36^{\wedge} \mathrm{N}$

where " $\mathrm{P}$ " is the sum of the given mathematical probabilities, and " $N$ " is the number of the main coding units to be used in each of the component columns Rows.

The triangular coding system contains a matrix of basic coding units which were arranged vertically and horizontally to form matrices that depend on the number of required mathematical variables (Figure-3). Since the cryptographic matrix consists of thirty-six variables, the user of the system can change the order of the matrix by assigning different numbers or letters for each of the designated variable. This will, in turn, increase the number of possibilities, by creating a unique key to solve the cryptographic matrix, to more than $1.06 \mathrm{x} 1056$. Health institutions can come up with unique and different matrix keys to store their information in a manner that is only accessible and identifiable by their own information technology systems.

The main function of the system is to store medical information based on the ICD-10, a medical classification list by the World Health Organization (WHO) where the amount of this information is based on the number of mathematical variables forming the matrices of basic coding units. The coding system is then identified using a digital scanner device that contains a non-transitory computer-readable medium storing the coding system, which is executable by a CPU system to identify three points in the upper half of each triangle, thus forming the basic coding units. It extracts the information from the triangular coding system and link the output to an internal or an external database.

\section{Discussion}

The ability to store and transfer encrypted medical information between different health institutions is of great importance. The current system, presented in this study, provides a simple process to store private medical information including patient's name, age, address, and medical history. This information can be then transferred electronically or printed without the fear of privacy or security violations. The current system may also be modified in the future to accommodate more variables and therefore store more advanced types of medical information.

\section{Acknowledgment}

The author would like to thank Ms. Nabila Qadri for her valuable support and encouragement during the preparation of the manuscript.

\section{Conflict of Interest}

This work has been granted a patent by King Abdulaziz City for Sciences and Technology in Saudi Arabia (IPC/SPO4678).

\section{References}

1. Cote RA, Robboy S. Progress in medical information management: Systematized Nomenclature of Medicine (SNOMED).1980; 243: 756-62.

2. Yan Y, Fung G, Dy JG, Rosales R. Medical coding classification by leveraging inter-code relationships. Proceedings of the 16th ACM SIGKDD International Conference on Knowledge Discovery and Data Mining; 2010.

3. Pereira S, Névéol A, Massari P, Joubert M, Darmoni S. Construction of a semi-automated ICD-10 coding help system to optimize medical and economic coding. MIE; 2006.

4. Al-Hariri A. Declaration of the cooperative health insurance companies and claims management companies and providers of health care services commence on the introduction of compulsory medical coding accredited. In: Services TCoH, editor. Riyadh: The Council Of Cooperative Health Insurance; 2012.

5. World Health Organization. International Statistical Classification of diseases and related health problems. 2009.

6. Libin B, Wachspress A. Method for transmitting medical information identified by a unique identifier barcode to a hospital. Google Patents; 2009.

7. Lv M, Hao YW, Gao Y. Applied research of bar code technology in high-value medical consumables management. Yiliao Weisheng Zhuangbei. 2012; 33: 119-21.

\section{*Correspondence to}

Bandar Ali Suliman

College of Applied Medical Sciences

Taibah University

Madinah

Saudi Arabia 